\title{
Combining hand grip strength with nutritional screening tools in elderly patients with chronic kidney disease
}

\author{
Anja Vukomanović ${ }^{1 *}$, Ivica Vrdoljak ${ }^{2}$, Ines Panjkota Krbavčić ${ }^{1}$, Tea Vrdoljak Margeta ${ }^{3}$, Martina Bituh $^{1}$
}

${ }^{I}$ Faculty of Food Technology and Biotechnology, University of Zagreb, Department of Food Quality Control, Laboratory for Food Chemistry and Biochemistry, Pierottijeva 6, 10000 Zagreb, Croatia

${ }^{2}$ Clinical Hospital Center Rijeka, Hospital Nutrition and Dietetics Service, Krešimirova 42, 51000 Rijeka, Croatia

${ }^{3}$ Clinical Hospital Center Rijeka, Department of Urology, Krešimirova 42, 51000 Rijeka, Croatia

*Corresponding author: avukomanovic@pbf.hr

\begin{abstract}
Malnutrition in vulnerable patient populations must be rapidly detected using techniques that are easy to incorporate into everyday clinical practice. The new recommendations defined the 7-point Subjective Global Assessment (SGA) as optimal for nutritional assessment in chronic kidney disease (CKD), while Geriatric Nutrition Risk Index (GNRI) demands additional examination in elderly. This study aimed to determine the accuracy of several concise tools used in the clinical practice and the correlation of this tools with functional method hand grip strength (HGS) in elderly patients with CKD. In this cross-sectional study, anthropometric and functional data for 50 elderly hemodialysis patients were analyzed using numerous survey-based tools for screening nutritional status (Malnutrition Screening Tool-MST, Nutritional Risk Screening 2002 - NRS2002, Malnutrition Universal Screening Tool - MUST, Mini Nutritional Assessment - MNA, GNRI), which we compared to the standard 7-point SGA nutritional assessment tool. The sensitivity, specificity, and accuracy of these tools for detecting malnutrition were compared with the standard by using receiver operating characteristic (ROC) curve analysis. 7-point SGA classified 36.6\% of participants as well nourished, and $63.4 \%$ as mildly to moderately malnourished, while the simplest alternative methods showed lower accuracy, classifying much higher proportions of participants as well nourished (MST, 92.0\%; NRS2002, 80.4\%). MNA had the highest accuracy based on receiver operating characteristic curves. HGS correlated moderately with 7-point SGA $(r=0.331), M N A(r=0.410)$, and GNRI $(r=0.320)$. Our small study suggests that MNA is the best tool for malnutrition risk screening in elderly with CKD. Combining HGS with concise tools, such as GNRI, may provide better results and unburden healthcare professionals.
\end{abstract}

\section{Keywords: chronic kidney disease, hemodialysis, malnutrition, nutritional status, screening}

\section{Introduction}

Malnutrition is one of the most common complications among individuals with chronic kidney disease (CKD), and it is a common cause of mortality and morbidity among patients on hemodialysis (Iorember, 2018). Regular nutritional screening and assessment of malnutrition are necessary because it often goes undiagnosed (Mueller et al., 2011). Nutritional screening has an important role in determining the need for further nutritional assessment, and ultimately in providing patients with appropriate nutritional care. Nutritional assessment of patients under the risk of malnutrition includes anthropometric, biochemical, clinical, dietary methods, and medical history; the most reliable assessments draw on combinations of these methods (Lee and Nieman, 2007).

The original Subjective Global Assessment (SGA) survey was the gold standard for detecting malnutrition in patients on hemodialysis (Detsky et al., 1987), but it is time-consuming and must be conducted by experienced medical staff (Keith, 2008). The need for a more precise assessment of the nutritional status of patients on hemodialysis resulted in changing the $\mathrm{ABC}$-scoring scale of the original SGA survey into a 7-point scale, generating the 7-point SGA (Churchill et al., 1996). New Kidney Disease Outcomes Quality Initiative (KDOQI) recommendations of the National Kidney Foundation (NKF) defined the 7-point SGA survey as a standard, valid, and reliable tool for assessing nutritional status in patients with stage 5 CKD (Ikizler et al., 2020). However, some tools require additional examination in elderly patients with CKD, highlighting the importance of researching the validity and reliability of Geriatric Nutrition Risk Index (GNRI) (Bouillanne et al., 2005) in elderly (Ikizler et al., 2020).

In addition to stated tools, many other survey-based tools have been designed as faster, simpler options in nutritional screening, such as Malnutrition Screening Tool (Ferguson et al., 1999) (MST), Nutritional Risk Screening 2002 (Reilly et al., 1995) (NRS2002), and Malnutrition Universal Screening Tool (Stratton et al., 2004) (MUST), while Mini Nutritional Assessment (Guigoz et al., 1994) (MNA) is the most extensive screening tool in this research. KDOQI defined all these tools, including GNRI, as screening tools (Ikizler et al., 2020) and some of these instruments have been validated only for specific patient populations (Kondrup et al., 2003). For example, NRS2002 can be used for in-patients, and MNA for the elderly, whereas MUST may be appropriate for any type of in- or outpatient.

The main goals of concise surveys for nutritional screening were directed towards practicality, time-saving, and diagnosing as many malnourished patients in hospitals, as it is recommended to performe it routinely. On the other hand, there is no defined standard method for nutritional screening in CKD and hemodialysis population (Ikizler et al., 2020); experienced medical professionals choose tools by their intuition. Current gaps and suggestions for future research (Ikizler et al., 2020) led us to undertake our comparison of concise nutritional screening surveys in the elderly population with CKD undergoing hemodialysis to determine if less complex tools will give reliable results, and if the complexity of tools represents an important factor. Also, we examined the application of the GNRI index, which demanded more additional research in the stated population. For the reference standard in tool evaluation we used the 7-point SGA, which was established as the reliable tool for assesing nutritional status in hemodialysis patients (Ikizler et al., 2020). Furthemore, reliable nutritional assessment tool was used as the reference in another study comparing nutritional screening tools in hemodialysis population (Yamada et al., 2008), what justified the usage 
of 7-point SGA in our research.

At the same time, we reasoned that we might improve the screening of nutritional status by complementing all survey-based tools with a functional test of hand grip strength (HGS), that can be used as indicator of protein-energy and functional status (Ikizler et al., 2020). Most hemodialysis patients are elderly with reduced muscle mass and strength, and HGS can predict all-cause mortality (Hwang et al., 2019; Vogt et al., 2016) and frailty (Johansen et al., 2019) in these patients. Therefore, we compared the sensitivity and specificity of various tools against 7-point SGA as the reference, and defined which tools are the most appropriate for nutritional screening in hemodialysis patients. Furthermore, we examined which tools correlated with HGS to propose a combination of methods to most reliably screen the nutritional status of elderly patients with CKD.

\section{Materials and methods}

\section{Participants}

This cross-sectional study was conducted at General Hospital „Dr. Josip Benčevićc in Slavonski Brod, Croatia. There were 97 patients in the hospital hemodialysis department, of whom 50 elderly patients aged 65 to 84 years were enrolled in the study. Exclusion criterias were age under 65 years, dementia or refusal to participate in the study. All patients underwent 4-5 hours of hemodialysis three times a week. Hemodialysis was performed using bicarbonate solution on high- and low-permeability polysulfonate dialyzers with standard rates of blood and dialysate flow. The study was conducted in accordance with the principles of the Declaration of Helsinki, and the study protocol was approved by the Ethics Committee of General Hospital „Dr. Josip Benčević". All participants signed written consent after being informed of the objectives of the study. Patients were assured that their data would be protected and anonymized for publication.

\section{Anthropometric assessment}

The following anthropometric measurements were taken approximately $30 \mathrm{~min}$ after hemodialysis as quickly as possible to minimize disturbance to the patient: body height, body weight, mid-upper arm circumference (MUAC) and calf circumference (CC) (Lee and Nieman, 2007). Weight $(\mathrm{kg})$ was measured using a standard clinical balance when participants

Table 1. Hand grip strenght (HGS) stratified by gender and body mass index (BMI) quartiles in order to define the risk of frailty (Fried et al., 2001)

\begin{tabular}{|l|c|}
\hline \multicolumn{1}{|c|}{ BMI quartiles according to gender } & Cut-off values for HGS (kg) \\
\hline Men & $\leq 29.0$ \\
\hline$\leq 24.0$ & $\leq 30.0$ \\
\hline $24.1-26.0$ & $\leq 30.0$ \\
\hline $26.1-28.0$ & $\leq 32.0$ \\
\hline$>28.0$ & \\
\hline Women & $\leq 17.0$ \\
\hline$\leq 23.0$ & $\leq 17.3$ \\
\hline $23.1-26.0$ & $\leq 18.0$ \\
\hline $26.1-29.0$ & $\leq 21.0$ \\
\hline$>29.0$ & \\
\hline
\end{tabular}

were barefoot, and height $(\mathrm{cm})$ was measured using a fixed stadiometer. Body weight at various times before enrollment in this study ( 2 weeks as well as 1, 2, 3, and 6 months) was collected from medical records. Body mass index (BMI) was calculated. MUAC was the average of three measurements on the nondominant arm, and $\mathrm{CC}$ was measured on the left calf. Both measurements were taken using inelastic tape while muscles were relaxed.

\section{HGS}

At 30 min after hemodialysis, HGS in the dominant hand was measured using a dynamometer (Seca, Germany) while participants sat in a angle, and the forearm and wrist positioned neutrally (Lee and Nieman, 2007). Participants were instructed to use as much pressure as possible. Measurements $(\mathrm{kg})$ were repeated three times and averaged HGS results were used to define if patients were under the risk of frailty based on an 8-level scale that takes into account gender and BMI (Table 1) (Fried et al., 2001).

\section{Blood biochemistry}

Blood samples were taken from participants once a month and analyzed in the Biochemical Laboratory of the same hospital. Blood samples for biochemical analyses were taken at the same period when anthropometric, functional parameters, assessment and screening tools were conducted. Serum concentrations of urea, creatinine, iron, unsaturated iron binding capacity (UIBC), total iron binding capacity (TIBC), sodium, potassium, calcium, phosphorous, total protein, albumin and cholesterol were determined using standard methods (Flegar Meštrić et al., 2000; Thomas, 1998; Tietz, 1987, 1995).

\section{Nutritional assessment and screening tools}

The 7-point SGA (Christensson et al., 2002) was considered the standard tool for nutritional assessment, classifying parcitipants as „well nourished“, „mildly to moderately malnourished“ or „severely malnourished“. Furthermore, all participants were screened using Geriatric Nutrition Risk Index (GNRI) (Bouillanne et al., 2005), Malnutrition Screening Tool (MST) (Ferguson et al., 1999), Nutritional Risk Screening instrument (NRS2002) (Reilly et al., 1995), Malnutrition Universal Screening Tool (MUST) (Stratton et al., 2004) and the neutral position with the shoulders adducted, elbows flexed at a $90^{\circ}$ 
complete version of Mini Nutritional Assessment (MNA) (Guigoz et al., 1994). All tools were completed by experienced medical staff and scored according to cut-off values described by the survey developers.

\section{Statistical analysis}

Data were analyzed using SPSS 17.0 (IBM, Chicago, IL, USA) and Excel 2013 (Microsoft, Seattle, WA, USA). Differences were considered significant if associated with $p<0.05$. Continuous data showing a normal distribution were reported as mean $\pm \mathrm{SD}$, while non-normal distribution was reported as median $\pm \mathrm{SEM}$. Differences between two age groups were assessed for significance using the independent samples t-test and Man-Whitney U test. Spearman rank correlation analysis was used for determining correlation between HGS and nutritional ssessment tools. The receiver operating characteristic (ROC) curves were generated. The accuracy of the screening tools for diagnosing malnutrition was assessed by calculating the area under the receiver operating characteristic curve (AUC), for which 7-point SGA served as the reference standard. AUC is 1.0 for a perfect test and no more than 0.5 for a useless test. Sensitivity, specificity and accuracy were determined using the cut-off points on the receiver operating characteristic curves that maximized the Youden index $J=$ maximum (sensitivity + specificity - 1) (Akobeng, 2007; Youden, 1950).

Table 2. Characteristics of the study population, stratified by age

\begin{tabular}{|c|c|c|c|c|}
\hline Characteristics & All $(n=50)$ & Age $<75(n=19)$ & Age $\geq 75(n=31)$ & $\mathrm{p}$ \\
\hline Age (years) & $76 \pm 12$ & $71 \pm 1$ & $79 \pm 1$ & $<0.001 *$ \\
\hline Duration of hemodialysis (months) & $25 \pm 8$ & $17 \pm 13$ & $39 \pm 11$ & 0.430 \\
\hline Weight $(\mathrm{kg})$ & $67.4 \pm 14.7$ & $68.8 \pm 17.8$ & $66.5 \pm 12.6$ & 0.603 \\
\hline Height $(\mathrm{cm})$ & $162.6 \pm 10.2$ & $167.6 \pm 2.65$ & $162.5 \pm 1.5$ & 0.549 \\
\hline $\mathrm{BMI}\left(\mathrm{kg} / \mathrm{m}^{2}\right)$ & $25.2 \pm 9.7$ & $25.2 \pm 0.8$ & $25.0 \pm 0.6$ & 0.928 \\
\hline $\operatorname{MUAC}(\mathrm{cm})$ & $25.1 \pm 2.9$ & $25.0 \pm 0.7$ & $25.65 \pm 0.54$ & 0.639 \\
\hline $\mathrm{CC}(\mathrm{cm})$ & $31.0 \pm 5.6$ & $30.8 \pm 0.8$ & $30.8 \pm 0.7$ & 0.984 \\
\hline HGS (kg) & $14 \pm 2$ & $15 \pm 3$ & $13 \pm 2$ & 0.992 \\
\hline Urea $(\mathrm{mmol} / \mathrm{L})$ & $7.2 \pm 2.8$ & $7.3 \pm 0.6$ & $6.7 \pm 0.5$ & 0.299 \\
\hline Creatinine $(\mu \mathrm{mol} / \mathrm{L})$ & $736 \pm 211$ & $749 \pm 192$ & $728 \pm 225$ & 0.729 \\
\hline Uric acid $(\mu \mathrm{mol} / \mathrm{L})$ & $321 \pm 56$ & $334 \pm 16$ & $329 \pm 9$ & 0.460 \\
\hline $\operatorname{Iron}(\mu \mathrm{mol} / \mathrm{L})$ & $8.6 \pm 0.4$ & $8.8 \pm 0.8$ & $9.1 \pm 0.5$ & 0.952 \\
\hline $\mathrm{UIBC}(\mu \mathrm{mol} / \mathrm{L})$ & $29 \pm 1$ & $31 \pm 3$ & $26 \pm 1$ & 0.238 \\
\hline TIBC $(\mu \mathrm{mol} / \mathrm{L})$ & $37.7 \pm 1.1$ & $39.2 \pm 2.1$ & $32.6 \pm 1.2$ & 0.281 \\
\hline Sodium $(\mathrm{mmol} / \mathrm{L})$ & $137 \pm 0$ & $137 \pm 1$ & $137 \pm 1$ & 0.632 \\
\hline Potassium $(\mathrm{mmol} / \mathrm{L})$ & $5.2 \pm 0.7$ & $5.2 \pm 0.1$ & $5.0 \pm 0.2$ & 0.208 \\
\hline Calcium $(\mathrm{mmol} / \mathrm{L})$ & $2.23 \pm 0.28$ & $2.30 \pm 0.05$ & $2.15 \pm 0.03$ & $0.003 *$ \\
\hline Phosphate (mmol/L) & $1.32 \pm 0.06$ & $1.59 \pm 0.16$ & $1.24 \pm 0.06$ & $0.006^{*}$ \\
\hline Total protein $(\mathrm{g} / \mathrm{L})$ & $66.24 \pm 5.71$ & $65.00 \pm 1.49$ & $64.00 \pm 0.94$ & 0.742 \\
\hline Albumin $(\mathrm{g} / \mathrm{L})$ & $33 \pm 0$ & $33 \pm 1$ & $32 \pm 1$ & 0.497 \\
\hline Cholesterol (mmol/L) & $4.3 \pm 1.0$ & $3.8 \pm 0.3$ & $4.5 \pm 0.2$ & 0.067 \\
\hline
\end{tabular}

BMI - body mass index; MUAC - mid-upper arm circumference; CC - calf circumference; HGS - hand grip strenght; UIBC - unsaturated iron binding capacity; TIBC - total iron binding capacity

*Significance based on the criterion $\mathrm{p}<0.05$ (independent samples t-test, Mann-Whitney U test). 


\section{Results and discussion}

The study included 50 elderly participants, of whom 19 (38\%) were 65 - 74 years old and 31 (62\%) 75 years or older. There was no difference between anthropometric and functional parameters according to participants age. The only observed differences between groups, except age, were in biochemical parameters in serum concentrations of calcium and phosphate (Table 2). Since differences between defined age groups were minimal, all participants were pooled in all subsequent analyses.

The UK Renal Association (Wright and Jones, 2009) recommends nutritional screening every 4-6 months for stable patients on hemodialysis and once per week for hospitalized patients, while patients at risk of malnutrition should be monitored more frequently. This highlights the need for an application of fast and simple nutritional screening tools.

Scores on the MUST, MNA, GNRI, and 7-point SGA were used to classify patients as being „,well nourished or low risk“ or being ,mildly to moderately malnourished or medium risk" or ,,malnourished or high risk". Scores on the MST and NRS2002 were used to classify whether patients were at risk of malnutrition or not (Figure 1). The cut-off scores for the different categories on the various tools came from the original survey reports.

Most participants were classified as well nourished by MST (94.0\%) and NRS2002 (83.7\%), compared to the 7-point SGA reference, which classified $36.6 \%$ of participants as well nourished, $63.4 \%$ as mildly to moderately malnourished and $0.0 \%$ as severely malnourished (Figure 1). GNRI classified $40.8 \%$ participants as well nourished, what did not deviate much from the standard, and showed the highest accuracy among other concise screening tools (Table 3).

Based on the three 7-point SGA categories of ,well nourished", „mildly to moderately malnourished" and „severely malnourished“, receiver operating characteristic curves were derived for every tool (Figure 2). All patients that 7-point SGA categorized as „,mildly to moderately malnourished“" or „severely malnourished“ were considered malnourished for the purposes of this analysis. MNA was the most accurate tool for malnutrition screening and showed moderate accuracy, what confirmed that more complex tools give more accurate results. Other tools showed low accuracy according to the receiver operating characteristic curves (Akobeng, 2007). NRS2002 and MST were the least accurate among the tools that we compared, and showed low accuracy along with MUST. Out of concise tools, GNRI gave the most accurate results (Table 3).

Previous research identified 7-point SGA as reliable nutritional predictor of clinical outcomes and mortality in hemodialysis patients (Borges et al., 2016; Sum et al., 2017). Although previous research showed that MNA was not as much reliable method as classic SGA in detecting malnutrition (Afsar et al., 2006), according to 7-point SGA as the reference standard, MNA was the most accurate screening tool and showed the highest sensitivity in this study (Table 3 ). This probably reflected the fact that incorporating a quantitative scale into classic SGA (Kalantar-Zadeh et al., 1999) affects the nutritional assessment process of hemodialysis patients.

Furthermore, GNRI showed low accuracy in nutritional screening of elderly patients on hemodialysis, indicating that preference in nutritional screening should be given to MNA. However, more factors must be taken into consideration when deciding which screening tool should be used in everyday clinical practice. The longer, more complex tool MNA was more accurate at classifying elderly participants as malnourished, indicating the need to collect data on a range of factors in order to accurately screen nutritional status of patients on hemodialysis. Consistent with this idea, only $2.0 \%$ of participants were underweight according to BMI, so relying on this index by itself could lead to substantial underdiagnosis of malnutrition (Cederholm et al., 2015). Despite the reported high prevalence of malnutrition among patients on hemodialysis, $38.0 \%$ of our subjects were overweight and $12.0 \%$ obese. This suggests that body weight and BMI may not necessarily be an adequate indicator of nutritional status in these patients and that combination of different parameters could provide better screening and assessement results (Iorember, 2018).

Although complete version of MNA was more accurate than other examined tools (Table 3), it is time consuming, demands obtaining patients anthropometric data and requires the capability of patients to self-estimate their dietary intake. The similarly concise MST, NRS2002 and MUST were the fastest and simplest tools in our experience and that of others (Stratton et al., 2004), but they showed low accuracy in our study (Table 3). This can be explained by all three tools featuring similar content about reduction of nutritional intake and unintentional weight loss (Ferguson et al., 1999; Reilly et al., 1995). GNRI was the most accurate concise tool for nutritional screening. This showed that combining biochemical and anthropometric parameters in nutritional screening in hemodialysis patients gives more reliable results. In this case, albumin and body weight modified by using Lorentz formula were combined. Moreover, GNRI is effective in predicting nutritional status in dialysis patients (Kang et al., 2013) and can be conducted fastly and practically just by healhtcare professionals; nutritional screening can be carried out by using data from patients medical charts respectively. Another studies showed that GNRI had the highest accuracy among other screening tools, but when using MIS as the reference standard

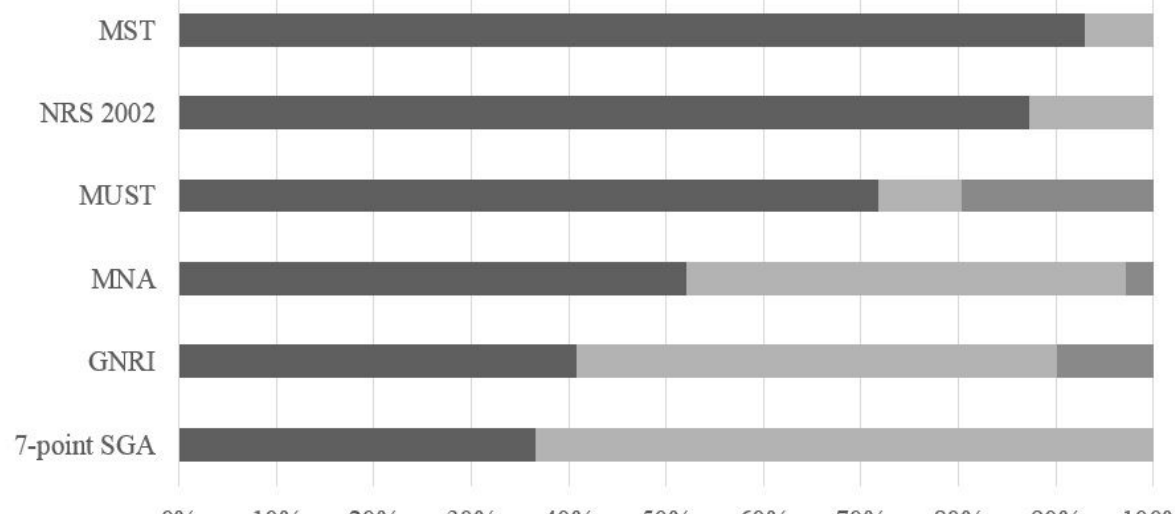

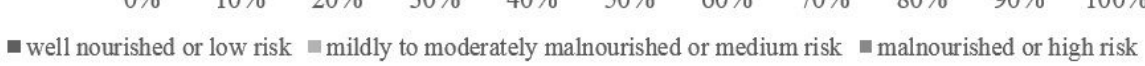

Figure 1. Classification of hemodialysis patients $(n=50)$ by nutritional status, obtained from using several nutritional screening tools. The standard was 7-point SGA tool. Cut-off values were taken from the original study reports. 

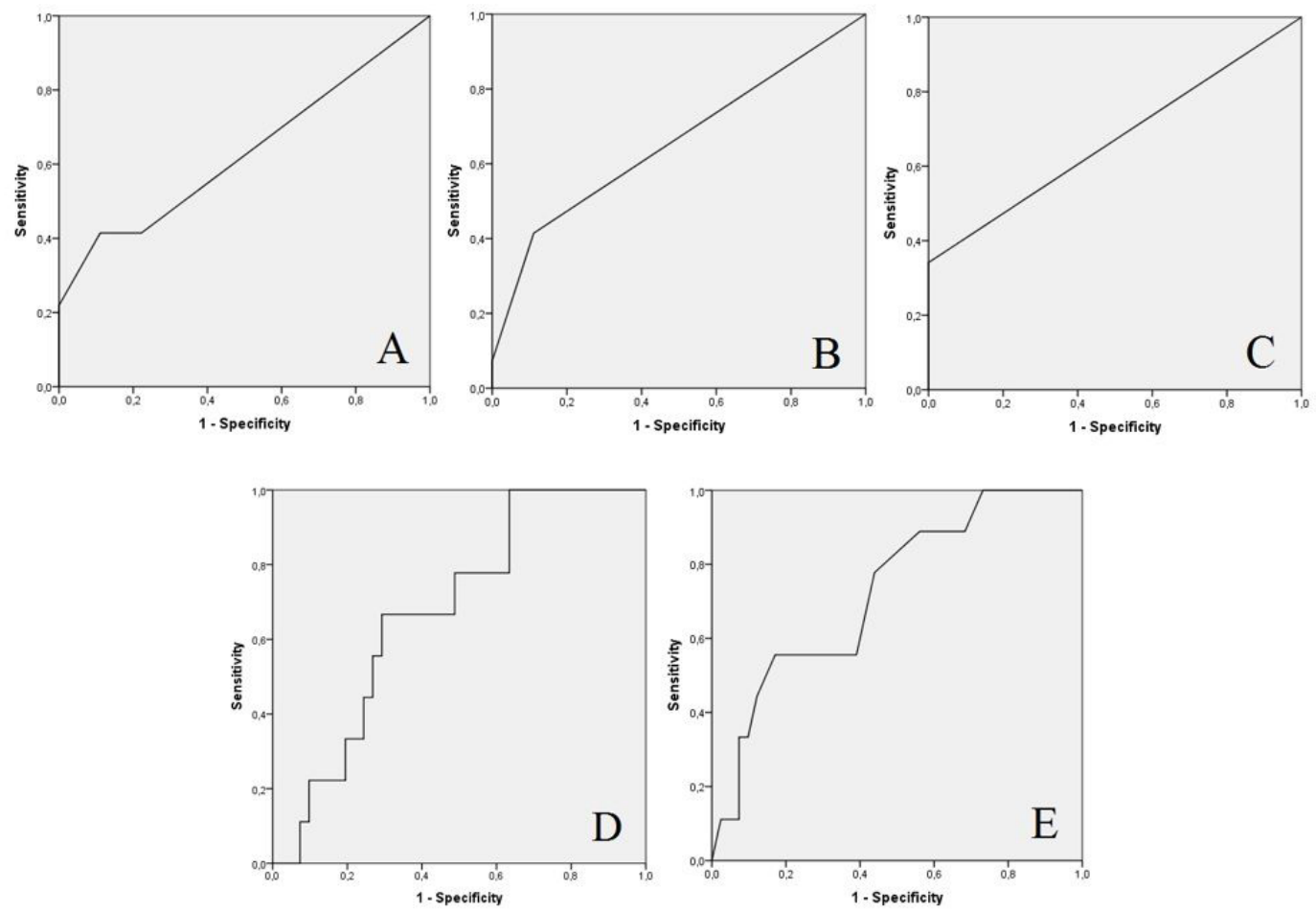

Figure 2. Receiver operating characteristic (ROC) curves quantifying the ability of various nutritional screening tools to diagnose malnourishment in hemodialysis patients, based on 7-point SGA as the reference standard: (A) NRS2002, (B) MST, (C) MUST, (D) GNRI, (E) MNA

Table 3. Sensitivity, specificity and accuracy of nutritional screening tools for diagnosing malnutrition in hemodialysis patients, based on receiver operating characteristic curves and 7-point SGA as the reference standard

\begin{tabular}{|l|c|c|c|}
\hline Tool & Sensitivity & Specificity & Accuracy \\
\hline NRS2002 & 0.415 & 0.889 & 0.631 \\
\hline MST & 0.415 & 0.889 & 0.656 \\
\hline MUST & 0.341 & 1.000 & 0.671 \\
\hline GNRI & 0.667 & 0.707 & 0.675 \\
\hline MNA & 0.889 & 0.439 & 0.728 \\
\hline
\end{tabular}

(Yamada et al., 2008).

Furthermore, we explored whether HGS could be a rapid way to improve the reliability of these concise and survey-based nutritional screening tools. HGS is often used in clinical practice to assess physical condition and even nutritional status (Flood et al., 2014), so it has proved to be a good predictor of muscle quality and survival in hemodialysis patients (Hwang et al., 2019; Vogt et al., 2016; Yoda et al., 2012). In our study sample, Spearman rank correlation analysis showed that HGS correlated moderately with 7-point SGA $(r=0,331)$, GNRI $(r=0,320)$, and MNA $(\mathrm{r}=0.410)$; weakly with MUST $(\mathrm{r}=-0.200)$; and not at all with MST $(\mathrm{r}$ $=-0.002)$ and NRS2002 $(r=-0.061)$.

Most participants who were under the risk of frailty based on published HGS cut-offs (Fried et al., 2001) were classified as malnourished or potentially malnourished by examined tools (Figure 3). In malnourished patients defined by GNRI, $81.1 \%$ of patients were under the risk of frailty. For example, of 14 participants defined by the GNRI as ,mildly to moderately malnourished or medium risk“", 13 (92.9\%) were under rhe risk of frailty based on HGS. The nutritional status of these patients should be monitored more frequently to detect emerging malnutrition as soon as possible.

The agreement observed between HGS and nutritional screening tools in our study is consistent with a report that low muscle strength correlates more strongly than low muscle mass with malnutrition and protein-energy wasting (Isoyama et al., 2014). Our results suggested that HGS can complement nutritional screening tools in elderly patients on hemodialysis, what is consistent with previous work showing that a combination of HGS and other anthropometric and nutritional parameters can reliably assess the nutritional status of such patients (Garcia et al., 2013). Also, a combined approach may be more reliable than assessments based on only one method (Kondrup et al., 2003), what encouraged us to examine it's effectiveness in nutritional screening as well.

Highest correlation with HGS was showed by nutritional assessmen tool 7-point SGA and screening tools MNA and GNRI. In other words, HGS correlated better with tools that collect data on more components and therefore require longer time and skill to administer. The exception was once again GNRI, which moderately correlated with HGS despite it's concisity. This led us to recommend using HGS when applying timesaving GNRI to get more reliable results. Ultimately, HGS may be useful for screening elderly patients who may require immediate nutritional screening with survey- or index-based tools, or for following up after such a tool indicates the risk of malnutrition.

This small study complements the new recommendations and provides evidence that MNA is valid tool for nutritional screening of elderly patients on hemodialysis. Among the nutritional screening tools, MNA was the most accurate for elderly patients on hemodialysis, while faster surveys such as NRS2002 and MUST were less accurate when reliable 7-point SGA was used as the reference standard. The study further shows that HGS correlates with many nutritional screening tools and can be combined with them to provide a more complete and reliable screening. In this context, MNA may be the best tool to combine with functional methods when screening the nutritional status of elderly hemodialysis 


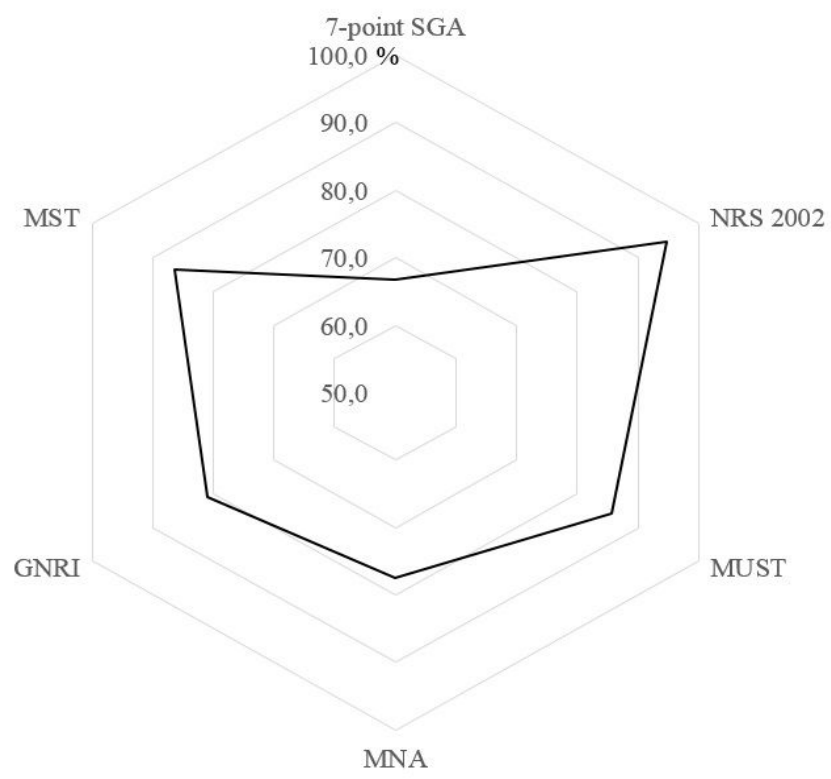

Figure 3. Proportions of hemodialysis patients classified as fragile based on hand grip strength who were also categorized as malnourished or potentially malnourished by the indicated nutritional screening tools. The concentric rings represent the indicated proportions.

patients. Furthermore, time-saving GNRI is the most accurate concise tool that could provide better results in screening nutritional status of elderly when combined with HGS.

\section{Conclusions}

It can be concluded that MNA can be used for nutritional screening of elderly hemodialysis patients. The advantage should be given to more complex tools, while simpler tools, such as GNRI, should be complemented by functional methods to get more reliable results. Screening tools should be further evaluated and future research should provide a standard and reliable tool for nutritional screening of elderly patients on hemodialysis. Future work should continue to explore rapid methods, alone and in combination, for accurate nutritional screening of this vulnerable patient population.

Acknowledgement: The authors would like to acknowledge the support of the medical and nursing staff as well as the participation of patients in this study.

Funding: The work was supported by General Hospital "Dr Josip Benčević", Slavonski Brod, Croatia.

Disclosure: The authors incertify that there is no conflict of interests.

\section{Abbreviations}

AUC - area under the receiver operating characteristic curve BMI - body mass index $\mathrm{CC}$ - calf circumference CKD - chronic kidney disease GNRI - Geriatric Nutrition Risk Index HGS - hand grip strength KDOQI - Kidney Disease Outcomes Quality Initiative MIS - Malnutrition Inflammation Score MNA - Mini Nutritional Assessment MST - Malnutrition Screening Tool MUAC - mid-upper arm circumference MUST - Malnutrition Universal Screening Tool NKF - National Kidney Foundation NRS2002 - Nutritional Risk Screening 2002 ROC - receiver operating characteristic curve SGA - Subjective Global Assessment TIBC - total iron binding capacity UIBC - unsaturated iron binding capacity

\section{References}

Afsar B., Sezer S., Arat Z., Tutal E., Ozdemir F. N., Haberal M. (2006) Reliability of Mini Nutritional Assessment in Hemodialysis Compared With Subjective Global Assessment. Journal of Renal Nutrition, 16(3) 277-282.

Akobeng A. K. (2007) Understanding diagnostic tests 3: Receiver operating characteristic curves. Acta Paediatrica, International Journal of Paediatrics, 96(5) 644-647.

Borges M. C. C., Vogt B. P., Martin L. C., Caramori J. C. T. (2016) Malnutrition Inflammation Score cut-off predicting mortality in maintenance hemodialysis patients. Clinical Nutrition ESPEN, 17 63-67.

Bouillanne O., Morineau G., Dupant C., Coulombel I., Vincent J. P., Nicolis I., Benazeth S., Cynober L., Aussel C. (2005) Geriatric Nutritional Risk Index: A new index for evaluating at-risk elderly medical patients. American Journal of Clinical Nutrition, 82(4) 777-783.

Cederholm T., Bosaeus I., Barazzoni R., Bauer J., Van Gossum A., Klek S., Muscaritoli M., Nyulasi I., Ockenga J., Schneider S. M., de van der Schueren M. A. E., Singer P. (2015) Diagnostic criteria for malnutrition - An ESPEN Consensus Statement. Clinical Nutrition, 34(3) 335-340.

Christensson L., Unosson M., Ek A. C. (2002) Evaluation of nutritional assessment techniques in elderly people newly admitted to municipal care. European Journal of Clinical Nutrition, 56(9) 810-818.

Churchill D. N., Wayne Taylor D., Keshaviah P. R. (1996) Adequacy of dialysis and nutrition in continuous peritoneal dialysis: association with clinical outcomes. Canada-USA (CANUSA) Peritoneal Dialysis Study Group. Journal of the American Society of Nephrology, 7(2) $198-207$.

Detsky A. S., Mclaughlin J., Baker J. P., Johnston N., Whittaker S., Mendelson R. A., Jeejeebhoy K. N. (1987) What is subjective global assessment 
of nutritional status? Journal of Parenteral and Enteral Nutrition, 11(1) 8-13.

Ferguson M., Capra S., Bauer J., Banks M. (1999) Development of a valid and reliable malnutrition screening tool for adult acute hospital patients. Nutrition, 15(6) 458-464.

Flegar Meštrić Z., Preden-Kereković V., Vrhovski-Hebrang D., Šurina B., Nazor A. (2000) Referentne vrijednosti biokemijskih i hematoloških sastojaka krvi i seruma zdravih osoba u dobi od 8 do 70 godina. Biochemia Medica, 10(1-2) 1-9.

Flood A., Chung A., Parker H., Kearns V., O'Sullivan T. A. (2014) The use of hand grip strength as a predictor of nutrition status in hospital patients. Clinical Nutrition, 33(1) 106-114.

Fried L. P., Tangen C. M., Walston J., Newman A. B., Hirsch C., Gottdiener J., Seeman T., Tracy R., Kop W. J., Burke G., McBurnie M. A. (2001) Frailty in Older Adults: Evidence for a Phenotype. The Journals of Gerontology Series A: Biological Sciences and Medical Sciences, 56(3) 146-156. Garcia M. F., Wazlawik E., Moreno Y. M. F., Führ L. M., González-Chica D. A. (2013) Diagnostic accuracy of handgrip strength in the assessment ofmalnutrition in hemodialyzed patients. E-SPEN Journal, 8(4) 181-186.

Guigoz Y., Vellas B., Garry P. J. (1994) Mini Nutritional Assessment: a practical assessment tool for grading the nutritional state of elderly patients. Facts and Research in Gerontology, 4(2) 15-59.

Hwang S. H., Lee D. H., Min J., Jeon J. Y. (2019) Handgrip Strength as a Predictor of All-Cause Mortality in Patients With Chronic Kidney Disease Undergoing Dialysis: A Meta-Analysis of Prospective Cohort Studies. Journal of Renal Nutrition, 29(6) 471-479.

Ikizler T. A., Burrowes J. D., Byham-Gray L. D., Campbell K. L., Carrero J. J., Chan W., Fouque D., Friedman A. N., Ghaddar S., Goldstein-Fuchs D. J., Kaysen G. A., Kopple J. D., Teta D., Yee-Moon Wang A., Cuppari L. (2020) KDOQI Clinical Practice Guideline for Nutrition in CKD: 2020 Update. American Journal of Kidney Diseases, 76(3) 1-107.

Iorember F. M. (2018) Malnutrition in chronic kidney disease. Frontiers in Pediatrics, 6161.

Isoyama N., Qureshi A. R., Avesani C. M., Lindholm B., Bárány P., Heimbürger O., Cederholm T., Stenvinkel P., Carrero J. J. (2014) Comparative associations of muscle mass and muscle strength with mortality in dialysis patients. Clinical Journal of the American Society of Nephrology, 9(10) $1720-1728$.

Johansen K. L., Delgado C., Kaysen G. A., Chertow G. M.., Chiang J., Dalrymple L. S., Segal M. R., Grimes B. A. (2019) Frailty Among Patients Receiving Hemodialysis: Evolution of Components and Associations With Mortality. Journals of Gerontology Series A Biological Sciences and Medical Sciences, 74(3) 380-386.

Kalantar-Zadeh K., Kleiner M., Dunne E., Lee G. H., Luft F. C. (1999) A modified quantitative subjective global assessment of nutrition for dialysis patients. Nephrology Dialysis Transplantation, 14(7) 1732-1738.

Kang S. H., Cho K. H., Park J. W., Yoon K. W., Do J. Y. (2013) Geriatric Nutritional Risk Index as a prognostic factor in peritoneal dialysis patients. Peritoneal Dialysis International, 33(4) 405-410.

Keith J. N. (2008) Bedside nutrition assessment past, present, and future: A review of the subjective global assessment. Nutrition in Clinical Practice, 23(4) 410-416.

Kondrup J., Allison S. P., Elia M., Vellas B., Plauth M. (2003) ESPEN guidelines for nutrition screening 2002. Clinical Nutrition, 22(4) $415-421$.

Lee R. D., Nieman D. C. (4th ed) (2007) Nutritional Assessment. McGraw-Hill, New York, USA.

Mueller C., Compher C., Ellen D. M. (2011) A.S.P.E.N. clinical guidelines: Nutrition screening, assessment, and intervention in adults. Journal of Parenteral and Enteral Nutrition, 35(1) 16-24.

Reilly H. M., Martineau J. K., Moran A., Kennedy H. (1995) Nutritional screening - Evaluation and implementation of a simple Nutrition Risk Score. Clinical Nutrition, 14(5) 269-273.

Stratton R. J., Hackston A., Longmore D., Dixon R., Price S., Stroud M., King C., Elia M. (2004) Malnutrition in hospital outpatients and inpatients: prevalence, concurrent validity and ease of use of the 'malnutrition universal screening tool' ('MUST') for adults. British Journal of Nutrition, 92(5) 799-808.

Sum S. S. M., Marcus A. F., Blair D., Olejnik L. A., Cao J., Parrott J. S., Peters E. N., Hand R. K., Byham-Gray L. D. (2017) Comparison of Subjective Global Assessment and Protein Energy Wasting Score to Nutrition Evaluations Conducted by Registered Dietitian Nutritionists in Identifying Protein Energy Wasting Risk in Maintenance Hemodialysis Patients. Journal of Renal Nutrition, 27(5) 325-332.

Thomas L. (1st ed) (1998) Clinical Laboratory Diagnostics: Use and Assessment of Clinical Laboratory Results. TH-Books, Renmark, Australia. Tietz N. W. (1st ed) (1987) Fundamentals of clinical chemistry (1st Edition). W.B. Saunders, Philadelphia, USA.

Tietz N. W. (3rd ed) (1995) Clinical Guide to Laboratory Tests, Irvine, USA.

Vogt B. P., Borges M. C. C., Goés C. R., de Caramori J. C. T. (2016) Handgrip strength is an independent predictor of all-cause mortality in maintenance dialysis patients. Clinical Nutrition, 35(6) 1429-1433.

Wright M., Jones C. (2009) Clinical Practice Guidelines: Nutrition in CKD. Available at: https://renal.org/sites/renal.org/files/nutrition-in-ckd-5thedition-1.pdf. Accessed: 27.07.2021.

Yamada K., Furuya R., Takita T., Maruyama Y., Yamaguchi Y., Ohkawa S., Kumagai H. (2008) Simplified nutritional screening tools for patients on maintenance hemodialysis. American Journal of Clinical Nutrition, 87(1) 106-113.

Yoda M., Inaba M., Okuno S., Yoda K., Yamada S., Imanishi Y., Mori K., Shoji T., Ishimura E., Yamakawa T., Shoji S. (2012) Poor muscle quality as a predictor of high mortality independent of diabetes in hemodialysis patients. Biomedicine and Pharmacotherapy, 66(4) 266-270.

Youden W. J. (1950) Index for rating diagnostic tests. Cancer, 3(1) 32-35. 